

\section{Understanding tomato plant metabolism for sustainable production}

Historically, crops have been domesticated without limiting their access to nutrients or water. Since these varieties did not need efficient nutrien absorption and distribution mechanisms, they are poorly adapted to a sustainable agricultural system which limits the need for excess fertiliser. Drs Laura Carrillo and Joaquin Medina of the Centre of Biotechnology and Genomics (CBGP) in Madrid, Spain, investigated the metabolic responses of tomacto plants to Minogan a expression, they establiched specific targets for future phat breeding. Itrogen is a crucial component for
plant growth and development.
lit has been supplemented by fertilisers since the 1950s; however, tis estimated that less than half the by the plant. This excessive amount of ertiliser leads to the release of harmfu greenhouse gases into the atmosphere and a significant eutrophication of our water systems. Increasing the efficiency by which plants use nitrogen would decrease the agricultural impact on climate change the agricultural impar
and biodiversity.

Dr Joaquin Medina, a senior principal Biotechnology and Genomics in Madid, Spain, and his colleague Dr Laura Carrillo, worked to identify key genes an metabolic pathways involved in water-

plants and crops like tomato (Solanum lycopersicum). Our current understanding of the genetic bases of plant responses to nitrogen limitation is based on studies of effects on the model plant Arabidopsis thaliana (small flowering plants related to mustard), as well as a few agricultura species, but the genetic components involved in nitrogen responses in vegetable crops like tomato has not been

\section{INVESTIGATING NITROGEN-}

\section{STRESS RESPONSES}

The researchers focused on the study of produced by the cell) and transcriptome (total RNA transcripts expressed in the cell) of different organs of tomato plants subjected to nitrogen limitation for different periods of time.

The tomato (Solanum lycopersicum L.) is one of the most globally important horticultural crops and has significant fertiliser ras been uuring the last decade, growth and fruit yield. However, the excess nutrients are usually washed of and pollute the natural environment, especially soils. By examining the metabolic and transcriptomic output of a plant deprived of a key nutrient, the can be revealed.
Using this approach, the research team examined genetic bases and identified key genes and metabolic pathways that are important to improve nitrogen use efficiency (NUE) that could be used in new tomato-breeding programs. NUE is an overalts aind nitroncompasses assimilation, and distribution throrge, the plant To improve NUE some of these aspects should be enhanced, even under limiting conditions.

To examine the effects of nitrogen limitation in this study, tomato plants were grown using sufficient nitrogen supplement $(8 \mathrm{Mm} \mathrm{N})$ and a reduced N supplementation (suboptimal) intended to mimic a more sustainable fertiliser supply ( $4 \mathrm{Mm} \mathrm{N}$ ). The team observed that the depleted group produced 1.7 times less biomass compared to the control plants. This was likely due to a eduction in chlorophyll production, leaf area, and photosynthetic output in the less efficient at papturing the plants were air, reducing the total carbon, as well as Nitrogen depletion also affected uptake of phosphorous and potassium in the leaves, indicating a significant link with both metabolic processes

\section{METABOLIC RESPONSES} TO SUBOPTIMAL

MTROGEN CONDITIONS

Limiting nitrogen avallability influenced the plants' carbon and nitrogen primary metabolism, which impacted the synthesis of sugars, organic acids, and amino acids. Specifically, the tomato plants deprived of nitrogen prioritised processes to maintain sucrose, glutamate, and asparagine (circal fornew tissue growh), and excess reduced their new shoot growth, and the source of sugar production was negatively storage sites. Sugar content increased in the roots but decreased in the leaves, indicating that most assimilated carbon was quickly exported from the leaf to sustain plant growth.

The researchers found that a reduction in sugar and amino acid production from the leaves led to a reduction in glutamic acid and GABA contents in fruit. Nitrogen depletion did not impact the fruit as much as the leaves, but it has the potential to change the flavour components due to the reduction in sugar and amino acid content, and this can significantly impact consumer experience.

Nitrogen depletion reduced total amino cid content and impacted the production of some amino acids over others, strategies. Integrative transcriptomic and metabolomic data analysis, using GWENA bioinformatic tools, found new links between specific gene clusters and primary metabolism compounds in leaves and roots. In leaves, genes involved in photosynthesis, metabolism, and nitroge sssimilation increased expression. Most notable of these were genes involved is amino acid biosynthesis and the Krebs Cycle, a key component in metabolism. The increased expression in roots involved genes associated with carbon and nitrogen metabolism and transport. Several of hese genes are correlated ind fhanges in nitrogen metabolic pathways.

\section{LIMITING NITROGEN AND}

ogen changed the expression of genes associated with both nitrogen and carbon metabolism and transport within the plants' organs. tanscriptome were observed in the eaves, indicating that nitrogen depletion has the greatest impact on photosynthes and amino acid metabolism. In addition to these, introgen-starved plants invoke alternative respiration and cyclic electron ransport processes to maintain their redox and ATPNADPH balance (the plant hinds alternative strategles to balance
Nutrient Responses

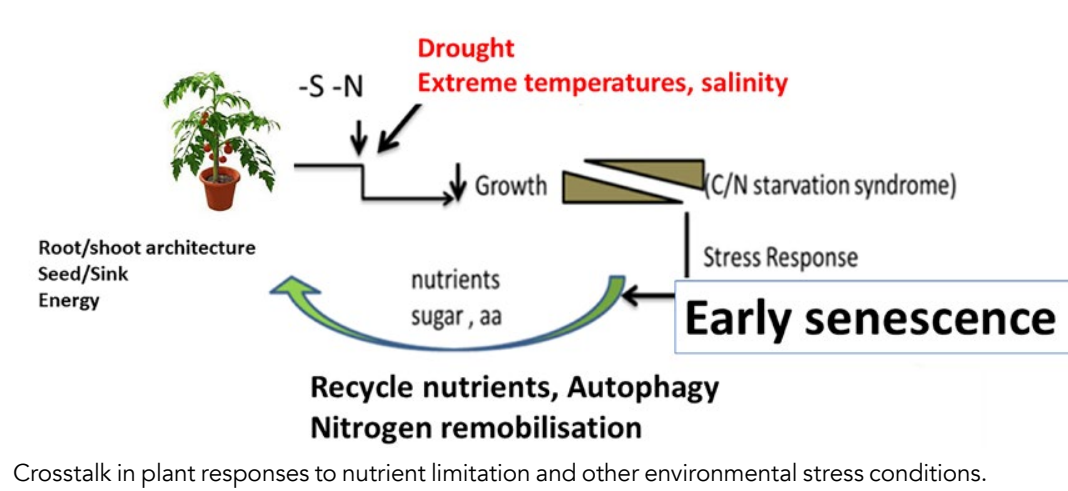

energy production). This is the first report of these alternative metabolic pathways being employed as a stress response in nitrogen-starved tomato leaves.

Nitrogen transporters are another nitrogen-efficient tomevoloping a components are important for nitroge distribution and uptake by different plant organs. The research team identified new nitrogen transporters in tomato. The expression levels of SINPF2.6 and SINPF2.11 genes were increased under nitrogen depletion in roots and leaves, and SINPF1.17, SINPF7.6, and SINPF7.3 were expressed especially in leaves. The different patterns of gene expression

levels might indicate that these genes play specific functions in nitrogen transport and recycling, especially from senescing leaves to different sink organs. Further study is needed to discover the exact functions and molecular buthey for optimal nitrogen efficiency.

\section{DENTIFYING KEY}

\section{TRANSCRIPTION REGULATORS}

Transcription factors requlate the expression (transcription) of genes, and they often regulate complex networks The research group uncovered new. transcriptional regulators that might be

Medina and Carrillo identified key metabolic pathways and genes that are important to improve nitrogen-use efficiency.

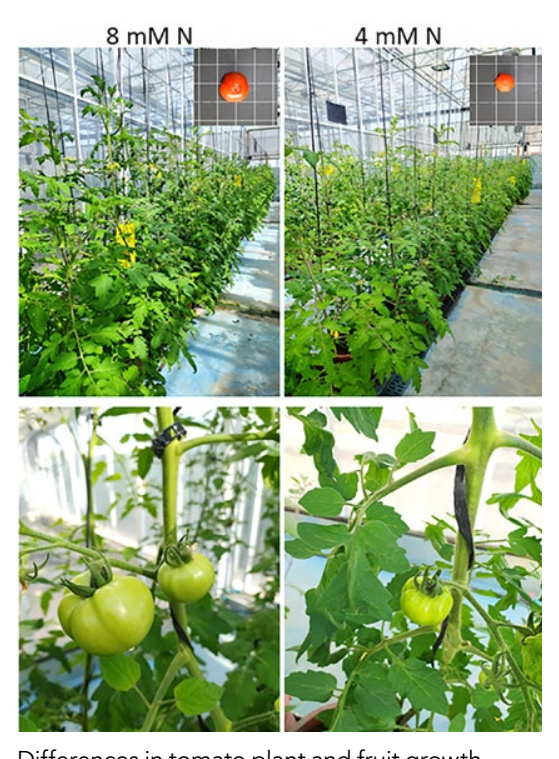

suboptimal nitrogen in tomato. Amon with high identity to TGA4, ARF8, HAT22, NF-YA5 and NLP9, which play key roles in nitrogen responses in Arabidopsis. Remarkably, only a few of the identified regulators are shared between roots and shoots, suggesting that common and
specific regulatory networks regulate introgen stress responses in tomato root and shoots.

This new knowledge of metabolic pathways, genes, and regulatory componentsinvolved in tomato nitrogenstress response will help to develop new plant varieties with improved NUE. Every little step helps reduce our dependence sustainable faliser and build an 

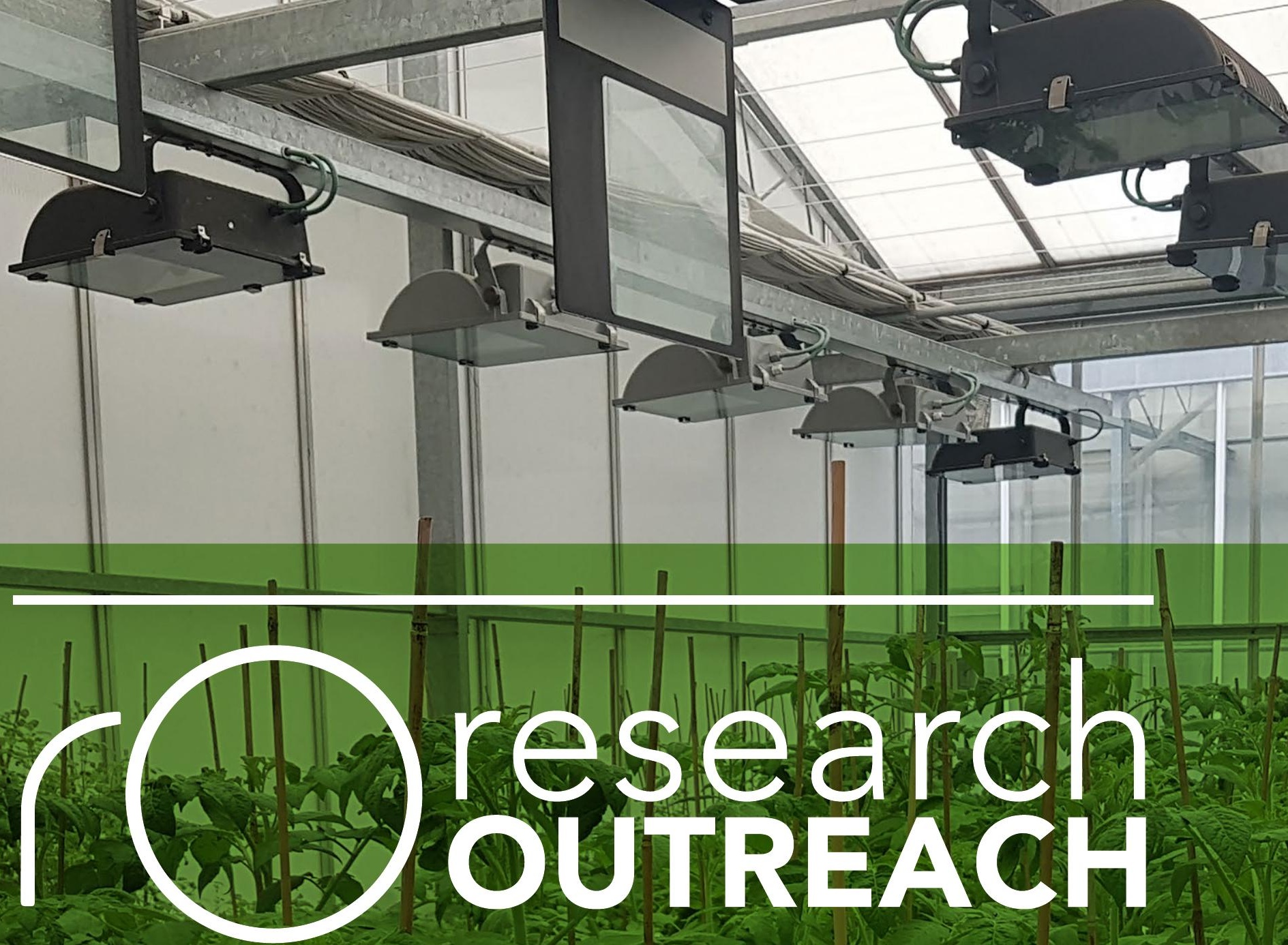

The public outreach magazine for the research community
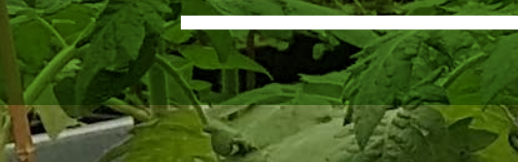

\section{researchoutreach.org}

Partnership enquinies: simpin@researchoutreach.orgy 